\title{
WG5 Highlights - Heavy Flavours
}

\section{Sebastian NAUMANN-EMME}

(Deutsches Elektronen-Synchrotron DESY, Hamburg, Germany)

E-mail: sebastian. naumann@desy.de

\section{Michel SAUTER}

(Heidelberg University, Heidelberg, Germany)

E-mail: michel.sauter@desy.de

\section{Antoni SZCZUREK}

(Institute of Nuclear Physics PAN, Kraków, and Rzeszów University, Rzeszów, Poland)

E-mail: antoni.szczurek@ifj.edu.pl

\begin{abstract}
We summarise the presentations that were given within the Heavy Flavour Working Group at the DIS2013 conference. Among the topics that were discussed are open heavy flavour production, heavy quarkonia, rare beauty decays, top-quark physics, and heavy-flavour production in heavyion collisions. The presentations were on theoretical methods as well as on recent experimental results from HERA, Tevatron, LHC, RHIC, and the B-factories at KEK and SLAC.
\end{abstract}

XXI International Workshop on Deep-Inelastic Scattering and Related Subject -DIS2013, 22-26 April 2013

Marseilles, France 


\section{Overview}

At this conference, several new developments related to heavy-flavour physics were presented from the theory side. We summarise these in Section 2. Furthermore, a huge amount of new experimental results was presented within the Heavy Flavour Working Group. In Section 3.1 the results on open charm and beauty production in $\gamma p$, ep and $p p$ scattering are summarised. Results on $J / \psi$ and $\Upsilon$ production and an update on the $\chi(4140)$ saga are given in Section 3.2. In Section 3.3 new results on heavy flavour hadroproduction are mentioned, while the measurements on top quark physics are discussed in Section 3.4. Finally, in Section 3.5 results on spectroscopy and beauty decays are summarised and in Section 3.6 an overview on the presented heavy flavour measurements with heavy ions is given.

Please see also [1,2] for the presentations given in the joint sessions with the Working Groups on "Structure Functions and Parton Densities" and on "QCD and Hadronic Final States".

Overall, the beautiful results that were presented reflect the impressive performance of the experiments and the progress achieved in the theoretical models, which generally describe the data remarkably well and are challenged by the measurements only in some cases.

\section{Theoretical aspects}

\subsection{General methods}

Recently, the publication of new data for charm production in semi-inclusive deep-inelastic scattering (DIS) and technical progress on the theory side motivated several analyses to determine the charm-quark mass from both DIS and hadronic data [3, 4]. Marco Guzzi discussed the charmquark mass impact on the CTEQ NNLO global analysis [6]. In their approach, the $\overline{\mathrm{MS}}$ mass $m_{c}\left(m_{c}\right)$ of the charm quark is extracted in the S-ACOT- $\chi$ heavy-quark factorisation scheme at the $\mathscr{O}\left(\alpha_{s}^{2}\right)$ accuracy. The obtained value of $m_{c}\left(m_{c}\right)$ is consistent with the world-average value. The best-fit values of $m_{c}\left(m_{c}\right)$ obtained via different approaches are shown in Fig. 1. The central result of $m_{c}=1.19_{-0.15}^{+0.08} \mathrm{GeV}$ is consistent with the PDG value of $1.275 \pm 0.025 \mathrm{GeV}$ within the errors. There is a small tendency in the different results to undershoot the PDG value which was attributed to missing $\mathscr{O}\left(\alpha_{s}^{3}\right)$ terms. Marco Guzzi and collaborators presented the systematic uncertainties of the extracted value by varying the DIS scale, the PDFs, $\alpha_{s}\left(M_{Z}\right)$, the $\chi^{2}$ definition, and a parameter $\lambda$ used in a generalized rescaling variable [7]. Varying $m_{c}$ leads to uncertainties on cross sections for different processes. Marco Guzzi showed how the Higgs gluon-gluon and $Z(l l)$ cross sections depend on the charm-quark mass in an interesting two-dimensional plot. The calculation had been performed within the NNLO accuracy.

Aleksander Kusina presented a new Hybrid Variable Flavour Number Scheme for heavyflavour production [8]. The approach, which was presented very recently in detail in [9] and seems to have practical character, incorporates the traditional Variable Flavour Number and Fixed Flavour Number schemes. In this new approach, one includes the explicit dependence on the number of active flavours in parton distribution functions and the strong coupling constant. One gets the freedom to choose the "optimal" number of flavours for each particular data set. Aleksander Kusina presented how such a fit can be implemented for both DIS and hadronic data. 


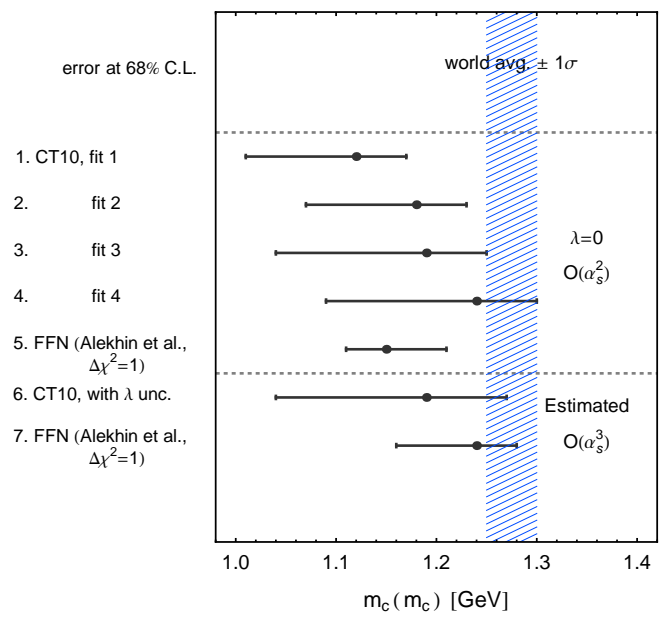

Figure 1: Best-fit values of $m_{c}\left(m_{c}\right)$ from different extractions.

It is quite interesting when the Standard Model may break down. One can answer this question based on the renormalization group (RG) analysis of the Standard Model running couplings such as the Higgs self-coupling $\lambda$. It is interesting if it stays positive up to the Planck scale. This would imply that the vacuum remains stable. Mikhail Kalmykov presented [10] results of a recent analysis of the electroweak contribution to the relation between pole and running masses of the top quark in the framework of the pure Standard Model. He argued that the use of the $\overline{\mathrm{MS}}$ running mass leads to better convergence properties than for the pole mass in the framework of the renormalization group equation. The quark anomalous dimension has two parts: the QCD and the electroweak contribution. Kalmykov and collaborators [11] obtained recently that the electroweak contribution is large and has opposite sign relative to the QCD contribution. The final result for the difference between pole and running mass, $m_{t}\left(M_{t}\right)-M_{t}$, is small (almost consistent with zero). Mikhail Kalmykov defined the optimal value for the RG matching scale as such when the radiative corrections to the matching conditions between the running coupling and the pole masses are minimal. Within the available accuracy, the IR-point lies in an interval between the Z-boson mass and the two masses of the $\mathrm{W}$ boson. The scale value is relevant for extractions of the Higgs self-coupling from the cross section. It is also useful for the construction of an effective Lagrangian.

\subsection{Production of open and hidden heavy flavours and decays of heavy-flavour mesons}

Rafał Maciuła discussed the production of charmed mesons at the LHC [12]. The presentation was based on Ref. [13]. The calculation, results of which were discussed during the conference, was performed in the framework of $k_{T}$-factorization. Different unintegrated gluon distributions (Kimber-Martin-Ryskin, Jung CCFM) were used in the calculation. Rafał Maciuła presented a detailed discussion of theoretical uncertainties due to the choice of the fragmentation and the renormalization scale, choice of the charm-quark mass, etc. The hadronization of charmed mesons was done with the help of the fragmentation-function technique. Inclusive differential distributions in the transverse momentum of charmed mesons were presented and compared to recent experimental data of ATLAS, CMS, ALICE and LHCb. The $k_{T}$-factorization approach is particularly good for 
correlation observables. Some examples of the comparison to the LHCb data are shown in Fig. 2. Good agreement is achieved. So far, there are no similar calculations in the framework of collinear factorization.


Figure 2: Invariant mass distribution of the $D^{0} \bar{D}^{0}$ system (left) and distribution in relative azimuthal angle between $D^{0}$ and $\bar{D}^{0}$ for different UGDFs, compared with the LHCb data.

Semileptonic decays of charm and bottom mesons are an efficient source of so-called nonphotonic electrons. This was achieved in the presented calculation with the help of decay functions for $D \rightarrow e^{ \pm}$and $B \rightarrow e^{ \pm}$fitted to CLEO and BaBar data. Inclusive differential distributions as a function of the transverse lepton momentum for several kinematic regions were presented and compared to recent results of the ALICE and CMS collaborations.

The situation at the LHC is quite different compared to that for RHIC or Tevatron as far as the production of charm quarks/antiquarks is considered. The quickly growing double-partonscattering cross section for the production of two $c \bar{c}$ pairs becomes comparable to that for the production of one pair $[14,15]$. Antoni Szczurek presented a first evaluation of several differential distributions for the production of $c \bar{c} c \bar{c}$ final states [16]. The double-scattering mechanism leads to the production of pairs of mesons: each containing c quarks or each containing $\bar{c}$ antiquarks. Some examples for the $\left(D^{0} D^{0}+\bar{D}^{0} \bar{D}^{0}\right)$ production are shown in Fig. 3. The calculations are compared with the LHCb experimental data [17]. The best description is obtained with the Kimber-MartinRyskin unintegrated gluon distribution. It was discussed that the contribution of single-parton scattering to the production of $c \bar{c} c \bar{c}$ is small, at least in the high-energy approximation. A better approach would require a full $2 \rightarrow 4$ calculation. Collinear approximation would be already a good start. It was emphasised that the double-parton scattering gives a large contribution to inclusive charmed meson spectra measured recently by the ALICE, ATLAS and LHCb experiments.

In the dominant production mechanism of heavy quarks the latter objects are produced radiatively in the final state. It was advocated already long ago by Stan Brodsky and collaborators [18] that the heavy quark can be a part of the non-perturbative proton structure. As heavy objects they would carry a relatively large momentum fraction of the proton. Direct photon production in association with a heavy quark is exactly one of the processes sensitive to the initial-state heavyquark distributions. This can be seen by analysing the leading-order contribution $g+Q \rightarrow \gamma+Q$. Tzvetalina Stavreva discussed an interesting problem of probing the intrinsic charm-quark content in the nucleon through direct photon-plus-heavy-quark production [19]. She presented a calculation which includes also higher-order contributions. A few models of the intrinsic charm were 

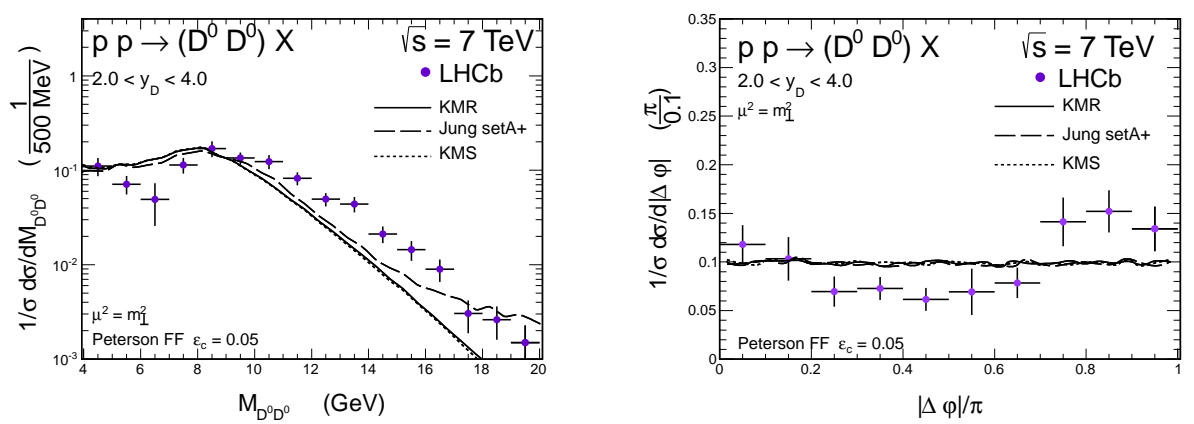

Figure 3: Invariant mass distribution of the $D^{0} D^{0}$ system (left) and distribution in relative azimuthal angle between $D^{0}$ and $D^{0}$ for different UGDFs, compared with the LHCb data.

discussed. They give quite a spread of predictions. The results of perturbative QCD (pQCD) calculations were compared to the D0 data. The distribution in the photon transverse momentum, associated with the charm, underestimates the D0 data at photon transverse momenta larger than $100 \mathrm{GeV}$. The resulting ratio "data to theory" is shown in Fig. 4. One can see some missing strength.

At the LHC, one probes in general much smaller proton momentum fractions $x$ than at the Tevatron. The forward rapidity region seems better in searches for "intrinsic charm". A prediction for future ATLAS measurements is shown in the right panel of Fig. 4. No quantitative information on the size of the exotic Fock component can be reached at present, based on the existing data and uncertainty of the theoretical calculations. Both experimental and theoretical improvements are needed to give a more definite answer.
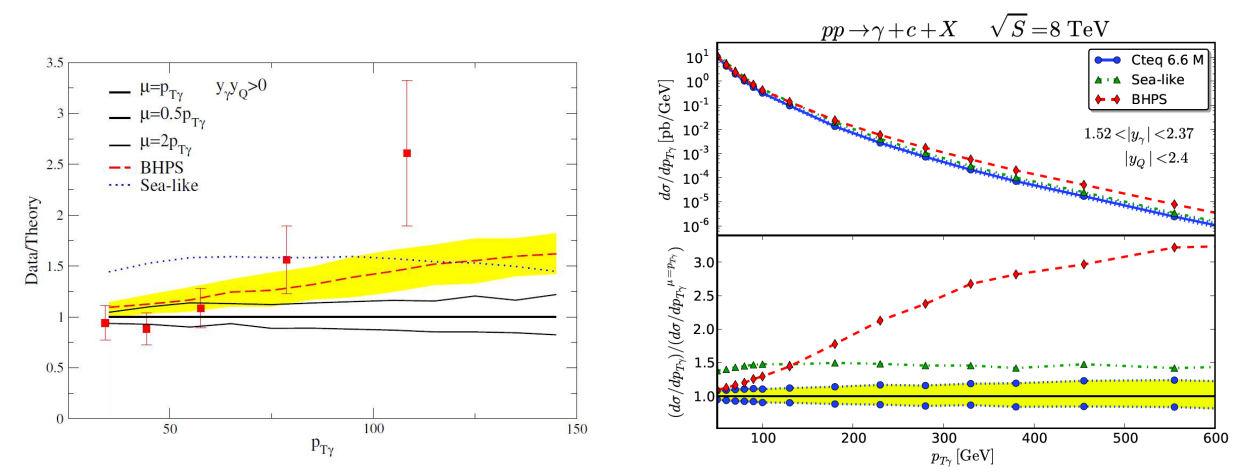

Figure 4: Data-over-theory ratio for $\gamma+c$ production (red squares) for the Tevatron conditions (left). Different theoretical predictions of the transverse momentum distribution of photons for LHC (right).

A recent $\mathrm{LHCb}$ measurement observed a large fraction of events with two $J / \psi$ mesons [21]. At present, there is no full theoretical understanding of the underlying reaction mechanism. Nikolai Zotov discussed the production mechanism of two $J / \psi$ mesons in proton-proton collisions at the LHC [22]. He focused on the interplay between the single-parton and double-parton mechanisms. It was shown that both mechanisms give similar contributions to the total cross section. However, a study of azimuthal correlations in rapidity between the two outgoing $J / \psi$ mesons allows for a separation between the dominant box contributions and the double-scattering one, driven at high energy by the $g g \rightarrow J / \psi g$ sub-process. The authors of Ref. [23] included also, for the first time, 
semi-diffractive contributions based on the $g g \rightarrow J / \psi J / \psi$ sub-process. Such processes also lead to a relatively large difference in rapidity between the two mesons. Fortunately, the corresponding contribution turned out to be much smaller than the double-parton-scattering one [23]. The relevant distribution in rapidity distance between the two $J / \psi$ mesons is shown in Fig. 5. One can observe that at the rapidity distance bigger than 3 units the double-scattering contribution should win over the other contribution. Practical predictions require dedicated studies including all experimental cuts.

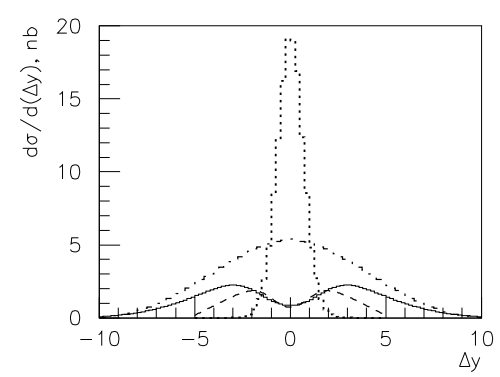

Figure 5: Distribution in rapidity difference between the two $J / \psi$ mesons. The dotted line corresponds to the box diagram, the dash-dotted line to the double-parton contribution. The one-gluon exchange contribution (solid curve) was multiplied by 1000, the two-gluon exchange contribution was multiplied by 25 .

The theoretical framework for computing rare radiative $B$ decays is QCD factorization [24]. Ruben Sandapen discussed rare radiative decays of $B$ mesons to vector mesons using distribution amplitudes calculated using holographic AdS/QCD [25]. AdS/QCD offers the advantage of avoiding end-point divergence problems. Together with Ahmady [26] they found that end-point divergences can be avoided when computing power-suppressed contributions in the heavy-quark limit. They have obtained a branching ratio $\bar{B}^{0} \rightarrow \rho^{0} \gamma$ compatible with the PDG as well as with recent Belle [27] and BaBar [28] data. They predicted also the correct amount of isospin breaking.

\section{Experimental aspects}

\subsection{QCD tests using open heavy flavour production}

The precise measurements of charm and beauty production cross sections in photoproduction ( $\gamma p$-scattering), deep-inelastic $e p$-scattering (DIS) and $p p$-scattering provide a powerful tool to test various aspects of $\mathrm{pQCD}$.

The H1 collaboration presented two new measurements of heavy-flavour photoproduction enriching heavy quarks with muons or electrons from semi-leptonic decays [29, 30]. In the muon analysis, beauty and charm jet cross sections are measured at moderate transverse momenta, whereas the electron analysis is focused to measure down to very low transverse momenta of the $b$-quark, i.e. a region of the phase space with the only available hard scale being the $b$-quark mass. In Fig. 6 a), the differential cross sections of these measurements and all currently available beauty photoproduction measurements at HERA are compared as a function of the $b$-quark momentum 
$p_{T}(b)$ to a massive NLO pQCD [31] prediction. It can be seen that the prediction agrees in general rather well with the data, which confirm each other over a wide range in $p_{T}(b)$.

In order to obtain a very precise determination of $\mathrm{F}_{2}^{\mathrm{c} \bar{c}}$, the component of the inclusive $F_{2}$ structure function with charm in the final state, the $\mathrm{H} 1$ and ZEUS collaborations combined their measurements of open charm production cross sections in DIS [4]. The combined data profit from a reduction of errors with respect to the individual measurements, due to improved statistical precision and due to cross-calibration of some systematic errors, reaching a total precision of about $5 \%$. This combined charm data together with the inclusive DIS cross sections from HERA allow in an NLO QCD analysis to study the influence of different heavy-flavour schemes on parton distribution functions and the value of the charm mass. The corresponding results are discussed in these proceedings in $[1,6]$. In addition, ZEUS presented new precise DIS cross sections from $D^{*}$ and $D^{+}$ mesons $[32,33]$. The $D^{*}$ and $D^{+}$data are each also used to extract $\mathrm{F}_{2}^{\mathrm{cc}}$ but are not yet contained in the combined data. In Fig. 6 b), a comparison of the three measurements is shown as a function of $x$ and $Q^{2}$, illustrating their agreement and the high precision.

a)

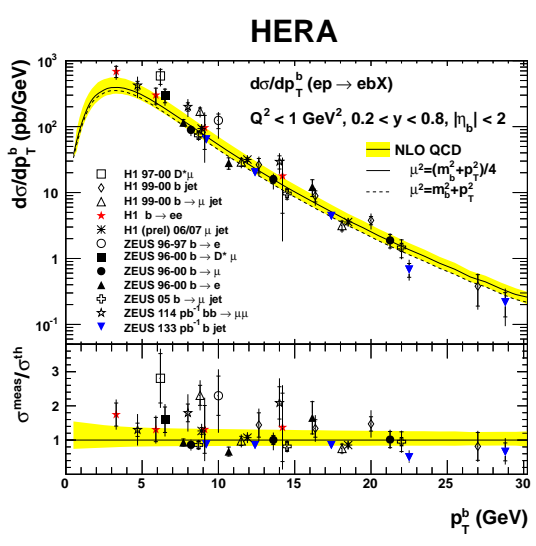

b)

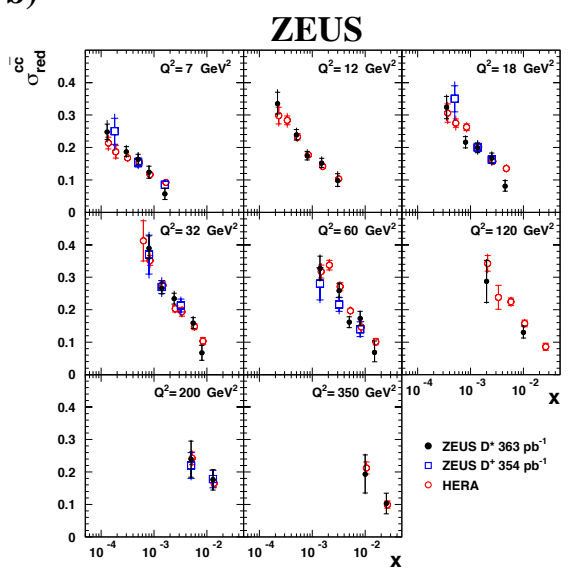

Figure 6: a) Compilation of beauty photoproduction cross section measurements at HERA as a function of the transverse momentum of the b-quark compared to a NLO pQCD calculation [31] (shaded band). b) Reduced charm cross sections from the combination of previous HERA results (empty circles), and from newer ZEUS measurements based on $D^{* \pm}$ (filled circles) and $D^{+}$(empty squares).

Various new measurements on the production of charm and beauty in $p p$-collisions at the LHC were reported by the LHCb, CMS and ATLAS collaborations, using different experimental techniques and covering different kinematic phase space ranges. The $\mathrm{LHCb}$ collaboration presented results on charm production using $D^{0}, D^{* \pm}, D^{+}, D_{s}^{+}$and $\Lambda_{c}^{+}$from secondary vertices [34] and beauty mesons with $B^{+}, B^{0}, B_{s}^{+}$[35]. Further, they reported on a measurement of the inclusive $c \bar{c}$ and $b \bar{b}$ cross section [36], as well as the forward-central $b \bar{b}$ production asymmetry [37]. The CMS collaboration showed results on $b \bar{b}$ angular correlations [38], results from the $\Lambda_{b}^{0}$-sector [39] and reported on the observation of $B_{c}^{+}$mesons in decays to $J / \psi \pi^{+}$or $J / \psi \pi^{+} \pi^{-} \pi^{+}$[40], whereas the ATLAS collaboration reported on the $b$-hadron production cross section from $D^{*} \mu$ final states [41] and the production cross section of $B^{ \pm}$mesons from decays into $J / \psi K^{ \pm}$[42]. All these measurements show agreement with the expectation from the Standard Model, and in general agree well 
with the NLO QCD calculations. As an example this is shown in Fig. 7 for results of $B^{+}$measurements as a function of $p_{T}\left(B^{+}\right)$compared to a $\mathrm{QCD}$ prediction for $B^{+}$production in the fixed-order plus next-to-leading logarithm approach (FONLL) [43].

a)

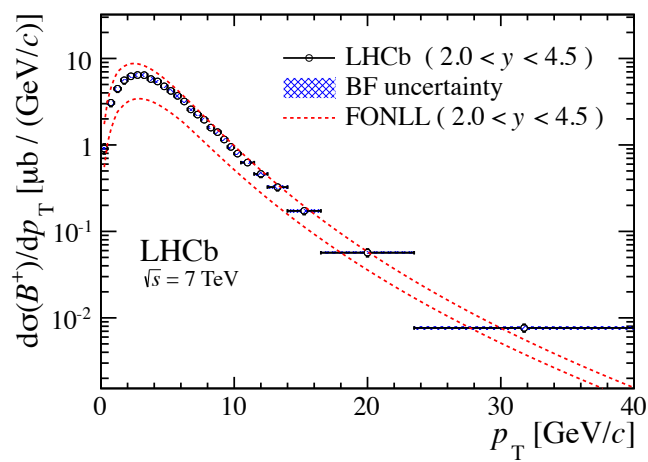

b)

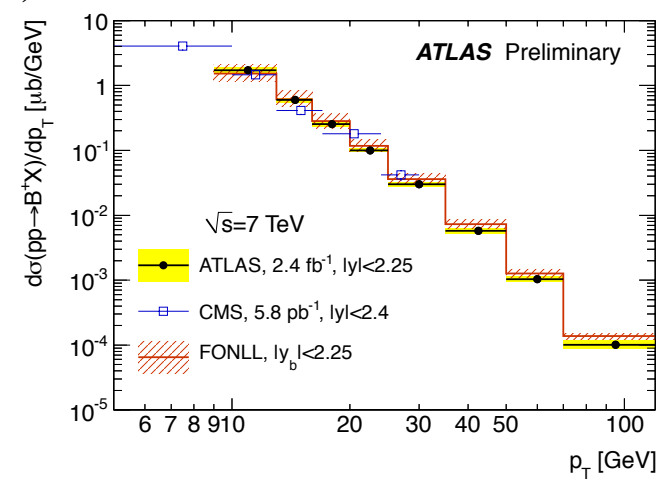

Figure 7: Differential cross sections of $B^{+}$production as a function of $p_{T}\left(B^{+}\right)$as measured by the LCHb collaboration a) and the ATLAS and CMS collaborations in a slightly different acceptance b). QCD predictions for FONLL [43] for $B^{+}$production are also given.

\subsection{Heavy-quarkonia}

\section{$J / \psi$ and $\Upsilon$ production}

The production of heavy-quarkonium also provides a powerful probe to test theory, since it involves both the production of heavy quarks and the modelling of hadronisation. In perturbative calculations, heavy quark pairs are either treated in colour-singlet (CS) or colour-octet (CO) states, whereas their relative contribution is treated differently in various models. The ZEUS collaboration published inelastic $J / \psi$ photoproduction cross sections [44] and compared the data to predictions of a full NLO CS+CO calculation and of a LO CS model calculation in the $k_{T}$-factorisation framework. Overall the $k_{T}$-factorisation framework provides a better description of the data, however for a better comparison with the data a reduction of theoretical uncertainty would be important. The CMS and ATLAS collaborations presented new measurements of $J / \psi$ and $\psi(2 s)$ production [45] and of $\Upsilon$ production [46, 47, 48] in $p p$ collisions. In [46] and [47], differential cross sections as a function of the transverse $\Upsilon$ momenta are measured for the states $\Upsilon(1 \mathrm{~s}), \Upsilon(2 \mathrm{~s})$ and $\Upsilon(3 \mathrm{~s})$ and compared to various theoretical models, finding that none provide an accurate description of the data. In [48], the polarisation of all three $\Upsilon$ states are investigated and no evidence for a strong polarisation is found. This result is consistent with previous data form the Tevatron [49], but is not described by theory predictions [50, 51]. Further, the ATLAS collaboration reported on the first observation of prompt $J / \psi$ meson production in association with a $W$ boson [52]: The observed signal is much higher than the predictions by the CS and CO models, leaving open the possibility for additional contributions to the observed rate in data. In addition, the ATLAS collaboration reported the observation of a new $\chi_{b}$ state in radiative transitions to $\Upsilon(1 s)$ and $\Upsilon(2 s)$ [53], and the CMS collaboration on the relative prompt production of $\chi_{c 1}$ and $\chi_{c 2}$ via their radiative decays $\chi_{c} \rightarrow J / \psi \gamma[54]$. 


\section{Exotic charmonium states}

The $\chi(4140)$ saga, present in the literature since a few years, got a an interesting update at this conference: The story was started earlier by the CDF collaboration which reported an evidence for a narrow near-threshold structure in the $J / \psi \phi$ mass spectrum in $B^{+} \rightarrow J / \psi \phi K^{+}$decays [55], which in an additional preliminary analysis was updated to a statistical significance of more than $5 \sigma$ [56]. In the CDF data, there is also room for a second structure at $4274 \mathrm{MeV}$. On the other hand, the Belle experiment did not find evidence for such a resonance in $\gamma \gamma \rightarrow J / \psi \phi$ events [57]. These results triggered a debate in the literature, as these structures are considered unusual and almost certain exotic. For a more complete discussion see the references given in [58]. The LHCb and CMS collaborations both inspected their data for a $\chi(4140)$-like resonance and reported their results [58, 59]: CMS confirms in a preliminary analysis a structure at $4148 \mathrm{MeV}$ with a significance of greater than $5 \sigma$ and also sees evidence for a second structure in the same mass spectrum. Contrary, the LHCb collaboration does not confirm a narrow structure near the threshold. The mass spectra of the results from CDF, CMS and LHb are presented in Fig. 8. From the discussions at this conference, no clear conclusion could be drawn. Additionally, both LHCb and CMS also presented new results on the nature of the $\chi(3872)$ state $[60,61]$.
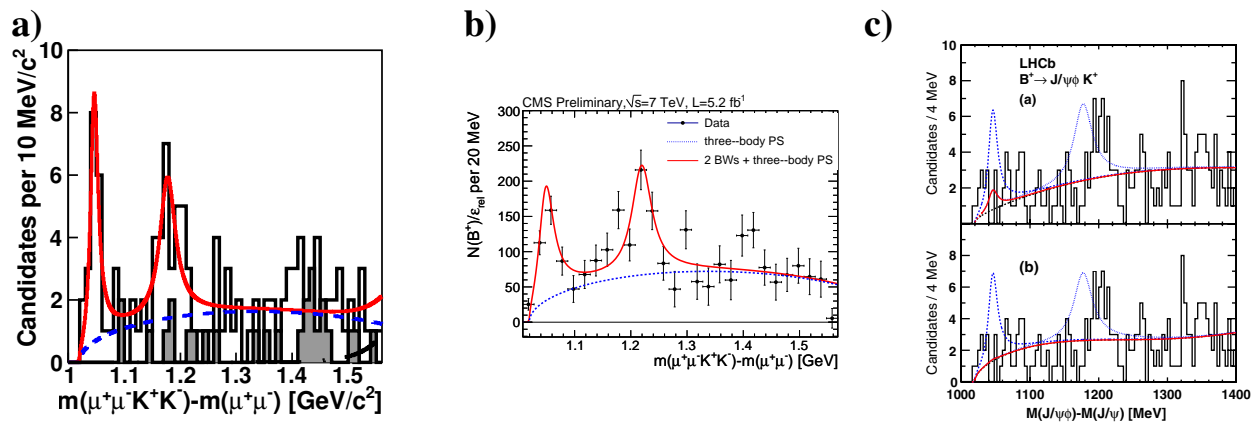

Figure 8: Distribution of the mass difference $M(J / \psi \phi)-M(J / \psi)$ in the $B^{+}$and $\phi$ mass windows, as determined by CDF a), CMS b) and $\mathrm{LHCb}$ c). The narrow peak at the threshold in a) is fitted under the assumption of a $\chi(4140)$ state and an additional resonance. b) The red line shows a fit under the hypothesis of two resonances. c) The dashed blue (dotted blue) line on top illustrates the expected signal yield from the CDF measurement, under different background assumptions (top and bottom).

\subsection{Heavy flavour in hadronic final states}

Another probe to test theory is the production of heavy-quark jets, which is composed of several mechanisms such as the heavy $Q \bar{Q}$ creation in the hard interaction, calculable in pQCD, or such as the parton-showering process, where non-perturbative corrections are required. The ATLAS collaboration presented an analysis [62] in which dijet heavy-flavour fractions are determined from multidimensional fits, and confronted with various predictions. The bottom-light flavour composition is found to be larger than the NLO or LO predictions, while other flavour compositions are reproduced by the predictions.

On the other hand, the fragmentation fractions of heavy quarks into heavy hadrons cannot be predicted but must be measured. Usually one assumes universality in the sense that the fragmentation fractions are independent of the production mechanism, i.e. are the same for $e^{+} e^{-}, \gamma p$ and 
$p p$ collisions. The ZEUS collaboration published precise measurements of charm fragmentation fractions in photoproduction $[63,64]$ to test this assumption and found good agreement with the $e^{+} e^{-}$data. Measurement [64] is based on excited $D_{1}$ and $D_{2}^{*}$ mesons and also contains results on masses of the neutral and charged states, the widths of the neutral states, and the helicity parameter of the $D_{1}(2420)^{0}$ meson.

\subsection{Top-quark physics}

ATLAS and CMS presented the status of their top-quark measurements in proton-proton collisions at $\sqrt{s}=7$ and $8 \mathrm{TeV}$. The focus in the corresponding session of this working group was on topquark properties, while measurements of cross sections for top-quark production were discussed in more detail within a dedicated session of WG1 ("Structure Functions and Parton Densities").

ATLAS presented a new study of heavy-flavour quarks produced in association with top-quark pairs [65], using the fudicial cross-section ratio $R_{\mathrm{HF}}=\sigma_{\mathrm{fid}}(t \bar{t}+\mathrm{HF}) / \sigma_{\mathrm{fid}}(t \bar{t}+j)$, where HF includes $b$ and $c$ but also $c \bar{c}$ and $b \bar{b}$, and $j$ refers to an additional jet of any flavour. Several scenarios beyond the SM predict additional HF in $t \bar{t}$ events. Moreover, $t \bar{t}+\mathrm{HF}$ is the main irreducible background for $t \bar{t}$-Higgs analyses. Using $4.7 \mathrm{fb}^{-1}$ of data at $\sqrt{s}=7 \mathrm{TeV}$, ATLAS observed $R_{\mathrm{HF}}=(7.1 \pm$ $\left.1.3(\mathrm{stat})_{-2.0}^{+5.3}(\mathrm{syst})\right) \%$, where the prediction at leading order and approximate next-to-leading order QCD is $(3.4 \pm 1.1) \%$ and $(5.2 \pm 1.7) \%$, respectively.

For the bottom-quark content in top-quark decays, CMS presented a new measurement of the relative branching fraction $\mathscr{R}=\mathscr{B}(t \rightarrow W b) / \mathscr{B}(t \rightarrow W q)$ with $q \in\{d, s, b\}$ [66]. The result based on $16.7 \mathrm{fb}^{-1}$ of data at $\sqrt{s}=8 \mathrm{TeV}$ is $\mathscr{R}=1.023_{-0.034}^{+0.036}$ (stat+syst), or $\mathscr{R}>0.945$ at $95 \%$ confidence level when requiring $\mathscr{R} \leq 1$. Assuming a unitary CKM matrix for three quark generations, this can be translated into $\left|V_{t b}\right|=1.011_{-0.017}^{+0.018}$ (stat+syst) or $\left|V_{t b}\right|>0.972$, respectively. These are the most precise measurement of $\mathscr{R}$ and the most stringent direct lower bound on $\left|V_{t b}\right|$ to date.

The V-A structure of the $W t b$ vertex can be tested via the polarisation of $W$ bosons produced in top-quark decays. The fraction of events containing $W$ bosons with longitudinal, left-handed or right-handed polarisation are referred to as $W$-helicity fractions. ATLAS and CMS had recently performed a preliminary combination of their measurements of the $W$-helicity fractions in $t \bar{t}$ events [67]. Additionally, CMS presented a first measurement of the $W$ polarisation in single-top events [68]. In all cases, the observed helicity fractions are in agreement with the predictions from NNLO QCD.

Measurements of the top-quark mass have almost reached the same precision at the LHC as at the Tevatron. The focus is thus shifting towards measurements with new, alternative methods as well as differential measurements, in order to gain a better understanding of the remaining systematic uncertainties and of top-quark mass definitions. CMS had recently released a study proving that the reconstructed mass as a function of a variety of kinematic variables in the $7 \mathrm{TeV}$ data-set is well modelled by the employed Monte-Carlo generators [69] and a first measurement of the $t$ vs. $\bar{t}$ mass difference from the $8 \mathrm{TeV}$ data-set [70], which yields $\Delta m(t, \bar{t})=-272 \pm 196$ (stat) \pm 122 (stat) GeV.

\subsection{Spectroscopy and beauty decays}

Results from the spectroscopy of heavy quarkonia and b hadrons as well as on rare beauty decays were presented by BaBar and Belle from the B-factories and from ATLAS, CMS, LHCb, 
and ATLAS, from the LHC and Tevatron, respectively. The discussed results represented the corresponding world-best measurement in many cases. Several results were new for this conference. Some examples will be given in the following.

The BaBar collaboration presented a new measurement of the $D^{*+}-D^{0}$ mass difference and of the natural line width of the transition $\mathrm{D}^{*}(2010)^{+} \rightarrow D^{0} \pi^{+}$[71]. The new result for the mass difference was approximately a factor 6 more precise than the world average, while the width was approximately 12 times more precise. D0 presented an updated measurement of the direct CP-violating charge asymmetry in $B^{ \pm} \rightarrow J / \psi K^{ \pm}$and $B^{ \pm} \rightarrow J / \psi \pi^{ \pm}$decays with $J / \psi \rightarrow \mu^{+} \mu^{-}$ in both cases using the full $10 \mathrm{fb}^{-1}$ data-set of the Tevatron Run-II [73]. Based on $3 \mathrm{fb}^{-1}$ of LHC proton-proton data collected at $\sqrt{s}=7$ and $8 \mathrm{TeV}$, LHCb presented the first observations of the decay $B_{c}^{+} \rightarrow \psi(2 s) \pi^{+}$with a significance of 5.2 standard deviations [74] and of the decays $B_{c}^{+} \rightarrow J / \psi(2 s) D_{s}^{+}$and $B_{c}^{+} \rightarrow J / \psi(2 s) D_{s}^{*+}$ with a significance larger than 9 standard deviations in each case [75]. The $B_{c}^{+} \rightarrow J / \psi(2 s) D_{s}^{+}$decay allowed $\mathrm{LHCb}$ to perform the most precise single measurement of the $B_{c}^{+}$to date. ATLAS presented an update of the $B_{S} \rightarrow J / \psi \phi$ analysis using $5 \mathrm{fb}^{-1}$ of the 2011 data, now including flavour tagging and thereby increasing the precision on the decay parameters further [76].

Overall, the presented result were compatible with the SM expectations. The largest tension was still related to the anomalous like-sign dimuon charge asymmetry observed by D0 [77].

\subsection{Heavy flavour from heavy ions}

The PHENIX and STAR collaborations presented the status of their measurements on open heavy flavour and quarkonia production in proton-proton, deuterium-gold, copper-copper, goldgold, and copper-gold collisions at RHIC. The ALICE collaboration summarized their heavyflavour measurements in proton-proton and lead-lead collisions at the LHC. In general, a reasonable description by $\mathrm{pQCD}$ predictions was found for the proton-proton results at these experiments, which also serve as a baseline for the measurements of cold and hot nuclear-matter (CNM and HNM, respectively) in heavy-ion collisions. Observations that are consistent with quark-gluonplasma effects had been made both at RHIC and the LHC, and heavy flavours appeared to be indeed good probes to study this state of matter. However, a yet better understanding of the effects in CNM was considered necessary before HNM properties could be thoroughly quantified. The 2013 proton-lead data from the LHC, which were still to be analyzed, might also shed further light on CNM effects like nuclear shadowing, gluon saturation, and the Cronin effect. The PHENIX and STAR experiments were looking forward to the data-taking in 2014 with upgraded detectors, which will be of particular benefit also for heavy-flavour measurements.

\section{References}

[1] C. Keppel, K. Lipka, P. Nadolsky, in these proceedings.

[2] A. Banfi, C. Glasman, B. Malaescu, in these proceedings.

[3] A. Martin et al., Eur. Phys. J. C70 (2010) 51, arXiv:1007.2624.

[4] H1 and ZEUS Collaborations, Eur. Phys. J. C 73 (2013) 2311 [arXiv:1211.1182 [hep-ex]].

[5] S. Alekhin et al.(2012), arXiv:1212.2355. 
[6] J. Gao, M. Guzzi and P. Nadolsky, proceedings to this conference.

[7] P. M. Nadolsky and W.-K. Tung, Phys. Rev. D79 (2009) 113014, arXiv:0903.2667.

[8] A. Kusina, F. I. Olness, I. Schienbein, T. Jezo, K. Kovarick, T. Stavreva and J.Y. Yu, proceedings to this conference.

[9] A. Kusina, F.I. Olness, I. Schienbein, T. Jezo, K. Kovarick, T. Stavreva and J.Y. Yu, arXiv:1306.6553.

[10] F. Jegerlehner, M. Kalmykov and B. A. Kniehl, proceedings to this conference.

[11] F. Jegerlehner, M.Yu, Kalmykov and B.A. Kniehl, Phys. Lett. B722 (2013) 123.

[12] R. Maciuła and A. Szczurek, proceedings to this conference.

[13] R. Maciuła and A. Szczurek, Phys. Rev. D87 (2013) 094022.

[14] M. Łuszczak, R. Maciuła and A. Szczurek, Phys. Rev. D85 (2012) 094034.

[15] R. Maciuła and A. Szczurek, Phys. Rev. D85 (2013) 074039.

[16] A. Szczurek and R. Maciuła, proceedings to this conference.

[17] LHCb Collaboration, J. High Energy Phys. 01 (2012) 141.

[18] S.J. Brodsky et al., Phys. Lett. B93 (1980) 451.

[19] T. Stavreva, proceedings to this conference.

[20] T. Stavreva and J.F. Owens, Phys. Rev. D79 (2009) 054017.

[21] LHCb Collaboration, Phys. Lett. B707 (2012) 52.

[22] N. P. Zotov et al., proceedings to this conference.

[23] S. P. Baranov, A. M. Snigirev, N. P. Szczurek, A. Szczurek and W. Schäfer, Phys. Rev. D87 (2013) 034035.

[24] S. W. Bosch and G. Buchalla, Nucl. Phys. B621 (2002) 459.

[25] M. Ahmady and R. Sandapen, proceedings to this conference.

[26] M. Ahmady and R. Sandapen, Phys. Rev. D87 (2013) 054013; M. Ahmady and R. Sandapen, arXiv:1305.1479.

[27] Belle Collaboration, Phys. Rev. Lett. 101 (2008) 111801.

[28] BaBar Collaboration, Phys. Rev. D78 (2008) 112001.

[29] H1 Collaboration, Eur. Phys. J. C 72 (2012) 2047 [arXiv:1205.2495 [hep-ex]].

[30] H1 Collaboration, Eur. Phys. J. C 72 (2012) 2148 [arXiv:1206.4346 [hep-ex]].

[31] S. Frixione, M. L. Mangano, P. Nason and G. Ridolfi, Adv. Ser. Direct. High Energy Phys. 15 (1998) 609 [hep-ph/9702287].

[32] ZEUS Collaboration, JHEP 1305 (2013) 097 [arXiv:1303.6578 [hep-ex]].

[33] ZEUS Collaboration, JHEP 1305 (2013) 023 [arXiv:1302.5058 [hep-ex]].

[34] LHCb Collaboration, Nucl. Phys. B 871 (2013) 1 [arXiv:1302.2864 [hep-ex]].

[35] LHCb Collaboration, arXiv:1306.3663 [hep-ex].

[36] LHCb COllaboration, LHCb-CONF-2013-002. 
[37] LHCb COllaboration, LHCb-CONF-2013-001.

[38] CMS Collaboration, CMS-PAS-BPH-10-019.

[39] CMS Collaboration, Phys. Lett. B 714 (2012) 136 [arXiv:1205.0594 [hep-ex]].

[40] CMS Collaboration, CMS-PAS-BPH-11-003.

[41] ATLAS Collaboration, Nucl. Phys. B 864 (2012) 341 [arXiv:1206.3122 [hep-ex]].

[42] ATLAS Collaboration, ATLAS-CONF-2013-008.

[43] M. Cacciari, S. Frixione, N. Houdeau, M. L. Mangano, P. Nason and G. Ridolfi, JHEP 1210 (2012) 137 [arXiv:1205.6344 [hep-ph]].

[44] ZEUS Collaboration, JHEP 1302 (2013) 071 [arXiv:1211.6946 [hep-ex]].

[45] CMS Collaboration, JHEP 1202 (2012) 011 [arXiv:1111.1557 [hep-ex]].

[46] ATLAS Collaboration, arXiv:1211.7255 [hep-ex].

[47] CMS Collaboration, arXiv:1303.5900 [hep-ex].

[48] CMS Collaboration, Phys. Rev. Lett. 110 (2013) 081802 [arXiv:1209.2922 [hep-ex]].

[49] CDF Collaboration, Phys. Rev. Lett. 108 (2012) 151802 [arXiv:1112.1591 [hep-ex]].

[50] B. Gong, J. -X. Wang and H. -F. Zhang, Phys. Rev. D 83 (2011) 114021 [arXiv:1009.3839 [hep-ph]].

[51] P. Artoisenet, J. M. Campbell, J. P. Lansberg, F. Maltoni and F. Tramontano, Phys. Rev. Lett. 101 (2008) 152001 [arXiv:0806.3282 [hep-ph]].

[52] ATLAS Collaboration, ATLAS-CONF-2013-042.

[53] ATLAS Collaboration, Phys. Rev. Lett. 108 (2012) 152001 [arXiv:1112.5154 [hep-ex]].

[54] CMS Collaboration, Eur. Phys. J. C 72 (2012) 2251 [arXiv:1210.0875 [hep-ex]].

[55] CDF Collaboration, Phys. Rev. Lett. 102 (2009) 242002 [arXiv:0903.2229 [hep-ex]].

[56] CDF Collaboration, arXiv:1101.6058 [hep-ex].

[57] Belle Collaboration, Phys. Rev. Lett. 104 (2010) 112004 [arXiv:0912.2383 [hep-ex]].

[58] LHCb Collaboration, Phys. Rev. D 85 (2012) 091103 [arXiv:1202.5087 [hep-ex]].

[59] CMS Collaboration, CMS-PAS-BPH-11-026.

[60] LHCb Collaboration, Phys. Rev. Lett. 110, 222001 (2013) [arXiv:1302.6269 [hep-ex]].

[61] CMS Collaboration, JHEP 1304 (2013) 154 [arXiv:1302.3968 [hep-ex]].

[62] ATLAS Collaboration, Eur. Phys. J. C 73 (2013) 2301 [arXiv:1210.0441 [hep-ex]].

[63] ZEUS Collaboration, arXiv:1306.4862 [hep-ex].

[64] ZEUS Collaboration, Nucl. Phys. B 866 (2013) 229 [arXiv:1208.4468 [hep-ex]].

[65] ATLAS Collaboration, arXiv:1304.6386 [hep-ex].

[66] CMS Collaboration, CMS-PAS-TOP-12-035.

[67] ATLAS and CMS Collaborations, ATLAS-CONF-2013-033, CMS-PAS-TOP-12-025.

[68] CMS Collaboration, CMS-PAS-TOP-12-020. 
[69] CMS Collaboration, CMS-PAS-TOP-12-029.

[70] CMS Collaboration, CMS-PAS-TOP-12-031.

[71] BaBar Collaboration, arXiv:1304.5009 [hep-ex].

[72] BaBar Collaboration, arXiv:1304.5657 [hep-ex].

[73] D0 Collaboration, arXiv:1304.1655 [hep-ex].

[74] LHCb Collaboration, Phys. Rev. D 87 (2013) 071103 [arXiv:1303.1737 [hep-ex]].

[75] LHCb Collaboration, Phys. Rev. D 87 (2013) 112012 [arXiv:1304.4530 [hep-ex]].

[76] ATLAS Collaboration, ATLAS-CONF-2013-039.

[77] D0 Collaboration, Phys. Rev. D 84 (2011) 052007 [arXiv:1106.6308 [hep-ex]]. 